\title{
The January 2015 outburst of a red nova in M 31
}

\author{
A. A. Kurtenkov ${ }^{1,8}$, P. Pessev ${ }^{2,7}$, T. Tomov ${ }^{3}$, E. A. Barsukova ${ }^{4}$, S. Fabrika ${ }^{4,15}$, K. Vida ${ }^{5}$, K. Hornoch ${ }^{6}$, E. P. Ovcharov ${ }^{1}$, \\ V. P. Goranskij ${ }^{9}$, A. F. Valeev ${ }^{4,15}$, L. Molnár ${ }^{5}$, K. Sárneczky ${ }^{5}$, A. Kostov ${ }^{8}$, P. Nedialkov ${ }^{1}$, S. Valenti ${ }^{10}$, S. Geier ${ }^{2,7}$,
} K. Wiersema ${ }^{11}$, M. Henze ${ }^{12,16}$, A. W. Shafter ${ }^{13}$, R. V. Muñoz Dimitrova ${ }^{8}$, V. N. Popov ${ }^{8}$, and M. Stritzinger ${ }^{14}$

1 Department of Astronomy, University of Sofia, 5 James Bourchier Blvd., 1164 Sofia, Bulgaria e-mail: al . kurtenkov@gmail.com

2 Instituto de Astrofísica de Canarias, 38200 La Laguna, Tenerife, Spain e-mail: peter.pessev@gtc.iac.es

3 Centre for Astronomy, Faculty of Physics, Astronomy and Informatics, Nicolaus Copernicus University, Grudziadzka 5, 87-100 Torun, Poland e-mail: tomov@umk.pl

4 Special Astrophysical Observatory, Nizhnij Arkhyz, Russia

5 Konkoly Observatory, Hungarian Academy of Sciences, 1121 Budapest, Konkoly Thege Miklós út 15-17, Hungary

6 Astronomical Institute, Academy of Sciences, 25165 Ondřejov, Czech Republic

7 Departamento de Astrofísica, Universidad de La Laguna, 38206 La Laguna, Tenerife, Spain

8 Institute of Astronomy and NAO, Bulgarian Academy of Sciences, 72 Tsarigradsko Chaussee Blvd., 1784 Sofia, Bulgaria

9 Sternberg Astronomical Institute, M.V. Lomonosov Moscow State University, Universitetskii pr. 13, 119992 Moscow, Russia

10 Las Cumbres Observatory Global Telescope Network, 6740 Cortona Dr., Suite 102, Goleta, CA 93117, USA

11 Department of Physics and Astronomy, University of Leicester, Leicester, LE1 7RH, UK

12 European Space Astronomy Centre, PO Box 78, 28692 Villanueva de la Cañada, Madrid, Spain

13 Department of Astronomy, San Diego State University, San Diego, CA 92182, USA

14 Department of Physics and Astronomy, Aarhus University, Ny Munkegade 120, 8000 Aarhus C, Denmark

15 Kazan Federal University, 420008 Kazan, Russia

16 Institut de Ciències de l'Espai (CSIC-IEEC), Campus UAB, C/Can Magrans s/n, 08193 Cerdanyola del Valles, Spain

Received 20 May 2015 / Accepted 28 May 2015

\section{ABSTRACT}

\begin{abstract}
Context. M31N 2015-01a (or M31LRN 2015) is a red nova that erupted in January 2015 - the first event of this kind observed in M 31 since 1988. Very few similar events have been confirmed as of 2015. Most of them are considered to be products of stellar mergers. Aims. Results of an extensive optical monitoring of the transient in the period January-March 2015 are presented.

Methods. Eight optical telescopes were used for imaging. Spectra were obtained on the Large Altazimuth Telescope (BTA), the Gran Telecsopio Canarias (GTC) and the Rozhen $2 \mathrm{~m}$ telescope.

Results. We present a highly accurate $70 \mathrm{~d}$ light curve and astrometry with a 0.05 " uncertainty. The colour indices reached a minimum of 2-3 d before peak brightness and rapidly increased afterwards. The spectral type changed from F5I to F0I in $6 \mathrm{~d}$ before the maximum and then to $\mathrm{K} 3 \mathrm{I}$ in the next $30 \mathrm{~d}$. The luminosity of the transient was estimated to be $8.7_{-2.2}^{+3.3} \times 10^{5} L_{\odot}$ during the optical maximum.

Conclusions. Both the photometric and the spectroscopic results confirm that the object is a red nova, similar to V838 Monocerotis.
\end{abstract}

Key words. novae, cataclysmic variables

\section{Introduction}

Red novae (or red transients) are a rare class of optical transients, reaching a peak luminosity equal to or higher than the brightest classical novae, but lower than supernovae. Many of them are best explained by binary star mergers (Tylenda \& Soker 2006) and therefore are often referred to as stellar mergers or "mergebursts" (Tylenda et al. 2011; Kochanek et al. 2014). Common observational properties during the first months of the outburst are the initial slow decline and a spectrum changing towards a late-type supergiant phase with colours shifting to the red.

The modern observational history of these events goes back to 1988 , when the transient M31-RV was detected by Rich et al. (1989) as an M0 supergiant variable with peak absolute $M_{\text {bol }} \sim-10$. A similar outburst in the Milky Way was observed in 1994, when V4332 Sgr changed its spectrum from K3 to M8-9

* Table 4 is available in electronic form at http://www . aanda.org during a three-month period (Martini et al. 1999). The eruption of V838 Mon in Feb. 2002 (Munari et al. 2002) reached an apparent magnitude of $V=6.7 \mathrm{mag}$ and was studied quite closely. The light curve showed three maxima and a consistent change of colours redwards as the spectrum reached an L supergiant phase at the end of the year (Evans et al. 2003). The resulting circumstellar light echo was used to obtain a distance estimate of $6.1 \pm 0.6 \mathrm{kpc}$, corresponding to a peak absolute magnitude $M_{V}=-9.8$ (Bond et al. 2003; Sparks et al. 2008). A bright event (peak $M_{R} \sim-12$ ), M85 OT2006-1, was reported by Kulkarni et al. (2007). Like other red novae, it showed a narrow $\mathrm{H} \alpha$ emission, a temperature decrease, and an infrared excess a few months after the eruption (Rau et al. 2007). V1309 Sco is another Milky Way red nova, discovered in 2008. Tylenda et al. (2011) have shown that the progenitor is a contact binary with a 1.4-day period, thereby adding a significant argument in favour of the stellar merger model for the cause of these outbursts. It has 
Table 1. Telescopes used for the monitoring of M31N 2015-01a.

\begin{tabular}{llccc}
\hline \hline Telescope & Observatory & Country & $\begin{array}{c}\text { Image scale } \\
{[\operatorname{arcsec} / \mathrm{px}]}\end{array}$ & Des. \\
\hline $6 \mathrm{~m}$ BTA & SAO RAS & Russia & 0.36 & BTA \\
$2.5 \mathrm{~m} \mathrm{NOT}$ & ORM & Spain & 0.19 & NOT \\
$2 \mathrm{~m}$ RCC & Rozhen NAO & Bulgaria & 0.74 & R2M \\
$1 \mathrm{~m} \mathrm{LCOGT}$ & McDonald & USA & 0.47 & LCOGT \\
$65 \mathrm{~cm}$ & Ondřejov & Czech Rep. & 1.05 & ONDR \\
$60 / 90 \mathrm{~cm}$ Schmidt & Konkoly & Hungary & 1.03 & KSCH \\
$50 / 70 \mathrm{~cm}$ Schmidt & Rozhen NAO & Bulgaria & 1.08 & RSCH \\
$50 \mathrm{~cm}$ Cassegrain & Uni Leicester & UK & 0.89 & UL50 \\
\hline
\end{tabular}

Notes. The last column contains the designations used in Table 4.

Table 2. List of reference stars.

\begin{tabular}{lccccc}
\hline \hline$\alpha(\mathrm{J} 2000)$ & $\delta(\mathrm{J} 2000)$ & $B$ & $V$ & $R$ & $I$ \\
\hline $00: 42: 08.9$ & $+40: 55: 33$ & 14.521 & 13.739 & 13.312 & 12.886 \\
00:42:12.6 & $+40: 54: 38$ & 14.792 & 14.267 & 13.996 & 13.699 \\
00:42:12.2 & $+40: 55: 49$ & 16.030 & 15.287 & 14.883 & 14.495 \\
00:42:05.3 & $+40: 53: 38$ & 16.876 & 15.915 & 15.381 & 14.822 \\
\hline rms of calibration & 0.015 & 0.007 & 0.008 & 0.015 \\
\hline
\end{tabular}

Notes. The magnitudes are calibrated by the Massey et al. (2006) catalogue using images from the $6 \mathrm{~m} \mathrm{BTA}$, the Rozhen $2 \mathrm{~m}$ and the Konkoly Schmidt telescopes.

been suggested that the first catalogued nova, CK Vul was also a "red transient" (Kaminski et al. 2015). A possible red nova in M101 was reported by Goranskij et al. (2015).

Here, we present a new addition to this class of objects, a red nova that erupted in M 31 in Jan. 2015. M31N 2015-01a was discovered by the MASTER-Kislovodsk system on 2015 Jan. 13 at $\sim 19.0 \mathrm{mag}$, unfiltered (Shumkov et al. 2015). It was incorrectly identified as a classical nova by Kurtenkov et al. (2015a) based on a strong $\mathrm{H} \alpha$ emission in the Jan. 16 spectrum that was also reported by Hodgkin et al. (2015). On Jan. 15 the object showed the spectral characteristics of an F5 supergiant (Fabrika et al. 2015). The transient became very bright, reaching a peak magnitude of $R \sim 15.1 \mathrm{mag}$ approximately on Jan. 22. It then proceeded to fade slowly and consistently turned redder. At the end of February, the colours had changed considerably, with the spectrum resembling that of a K supergiant. Subsequently, we announced that the transient is a red nova (Kurtenkov et al. 2015 b). Our supposition that the spectral evolution of the object is very similar to the observed in V838 Mon was confirmed by Williams et al. (2015, Fig. 2). The possible progenitor system has been discussed by Dong et al. (2015) and Williams et al. (2015).

\section{Observations and data reduction}

We performed imaging of M31N 2015-01a in Johnson-Cousins $B V R I$ filters (Table 1). The science frames were divided by flat fields after dark and bias subtraction.

Images of vastly different depths and fields of view (FOVs) were obtained. To perform all the photometry in a similar manner, we used four bright reference stars within 1.6' from the transient (Table 2). That way, systematic effects over large FOVs do not affect the results. This also allowed us to minimize the instrumental errors of the references. Three of those four stars were used for each frame. Massey et al. (2006) found systematic differences of about 0.1 mag between their magnitudes and those by Magnier et al. (1992), therefore we used the Massey catalogue to recalibrate the $B V R I$ magnitudes of the four references. Two
Table 3. Log of spectral observations.

\begin{tabular}{llccc}
\hline \hline Date & Telescope/Instrument & $R$ & Range $[\AA]$ & Exp. time [s] \\
\hline Jan. 15 & BTA/SCORPIO & 1000 & $4050-5850$ & 1500 \\
Jan. 16 & 2mRCC/FoReRo2 & 400 & $5500-7500$ & $4 \times 900$ \\
Jan. 17 & 2mRCC/FoReRo2 & 400 & $5500-7500$ & $5 \times 900$ \\
Jan. 21 & BTA/SCORPIO & 1000 & $4050-5850$ & 1800 \\
Feb. 21 & BTA/SCORPIO & 1000 & $3720-5530$ & 2700 \\
Feb. 22 & BTA/SCORPIO & 1000 & $5750-7500$ & 1800 \\
Feb. 24 & GTC/OSIRIS & 2500 & $4430-9090$ & $6 \times 180$ \\
\hline
\end{tabular}

Notes. The instruments are described by Jockers et al. (2000), Afanasiev \& Moiseev (2005), and Cepa (1998).

images per filter and between 18 and 30 stars per image were used for the recalibration. The peak signals of the four reference stars in these images are within the linear range of the detector. The results are presented in Table 2. Aperture photometry was made for all individual frames. The aperture radii we used were in the range of $1-1.5 F W H M$ of the Gaussian profiles of point sources. The results are presented in Table 4. Only the mean JD and median magnitude are given where several consecutive images are available.

Spectra of M31N 2015-01a were obtained with three telescopes - the $6 \mathrm{~m}$ Large Altazimuth Telescope (BTA) at the Special Astrophysical Observatory of the Russian Academy of Sciences (SAO RAS), the $10.4 \mathrm{~m}$ Gran Telescopio Canarias (GTC) at the Observatorio del Roque de los Muchachos (ORM), and the $2 \mathrm{~m} \mathrm{RCC}$ telescope at the National Astronomical Observatory Rozhen, Bulgaria (NAO) (Table 3). All the data reduction and calibrations were carried out with standard MIDAS ${ }^{1}$ (BTA) and IRAF $^{2}$ (2m RCC and GTC) procedures. The BTA and the GTC spectra were calibrated in relative fluxes using spectrophotometric standards G191-B2B, Hiltner 600, and Hz 2 (Oke 1990; Hamuy et al. 1992, 1994).

\section{Results and discussion}

\subsection{Astrometry}

Astrometric calibrations were made using both the PPMXL (Roeser et al. 2010) and the LGGS (Massey et al. 2006) catalogues. Astrometric solutions were obtained for five images from the $10.4 \mathrm{~m}$ GTC, $6 \mathrm{~m}$ BTA, and $1 \mathrm{~m}$ Las Cumbres Observatory Global Telescope (LCOGT) telescopes with an atmospheric seeing in the range of $1.3^{\prime \prime}-1.7^{\prime \prime}$. Separate calibrations were made using 20 relatively bright stars from the PPMXL catalogue and 16 fainter stars close to the transient from LGGS. From PPMXL we derived $\alpha, \delta(\mathrm{J} 2000)=00^{\mathrm{h}} 42^{\mathrm{m}} 08^{\mathrm{s}} .053,+40^{\circ} 55^{\prime} 01^{\prime \prime} .27$ with uncertainties of $\Delta_{\alpha} \sim 0.2^{\prime \prime}, \Delta_{\delta} \sim 0.1^{\prime \prime}$. The LGGS calibrations yielded $\alpha, \delta(\mathrm{J} 2000)=00^{\mathrm{h}} 42^{\mathrm{m}} 08^{\mathrm{s}} .065,+40^{\circ} 55^{\prime} 01^{\prime \prime} .33$ with $\Delta_{\alpha} \sim \Delta_{\delta} \sim 0.05^{\prime \prime}$.

\subsection{Photometry}

The light curve of M31N 2015-01a (Fig. 1) shows a distinct similarity to the V1309 Sco light curve during the first month of the outburst (Mason et al. 2010). The Jan. 21 datapoints are obtained shortly after the $B$-band and before the $R$-band maxima; we therefore assume that they coincide with the $V$-band maximum at $15.43 \mathrm{mag}$. The initial fading has been quite slow. The

\footnotetext{
1 See http://www.eso.org/sci/software/esomidas/

2 IRAF is distributed by the National Optical Astronomy Observatory, which is operated by the Association of Universities for Research in Astronomy (AURA) under a cooperative agreement with the National Science Foundation.
} 
A. A. Kurtenkov et al.: The January 2015 outburst of a red nova in M 31

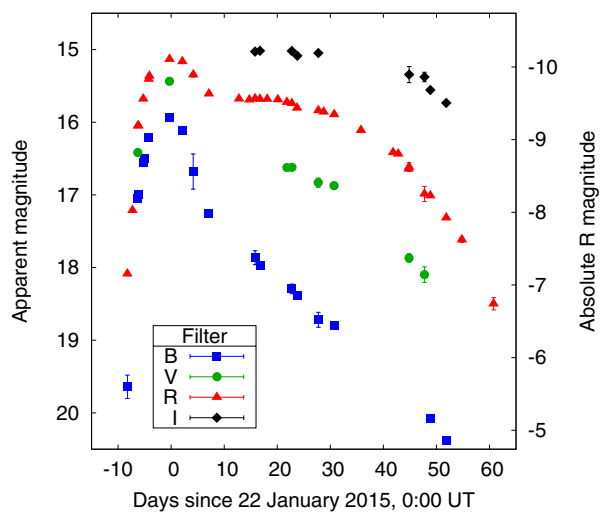

Fig. 1. Light curve of M31N 2015-01a in Johnson-Cousins BVRI filters. The zero-point moment roughly coincides with the $R$-band peak. The absolute magnitude scale (right) only applies to the $R$-band datapoints.

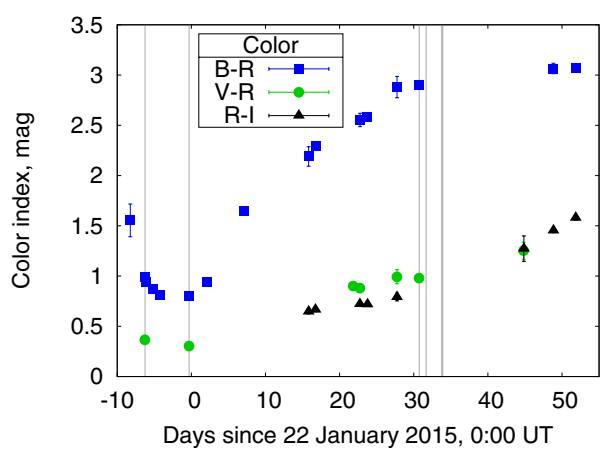

Fig. 2. Change of the observed colour indices. Vertical lines represent the times at which the BTA (thin) and GTC (thick) spectra were taken.

decline times by 2 mag after the maximum $t_{2}$ were $\sim 17 d, \sim 40 d$, and $\sim 50 \mathrm{~d}$ in $B, V$, and $R$ bands, respectively. The colours consistently shifted towards the red, for example, $B-R$ increased by $2.1 \mathrm{mag}$ in the 31 days after the maximum. The $R$ magnitude reached a plateau 0.6 mag below the maximum in $\sim 10 \mathrm{~d}$, but no rebrightening followed, as was the case with V838 Mon.

Figure 2 shows the change of colours. The colour temperature initially increased and reached a peak 2-3 d before the $R$-band maximum. It consistently decreased afterwards as the $B-R$ index increased by $\sim 2$ mag in one month.

\subsection{Spectroscopy}

The aim of obtaining the FoReRo2 spectra of M31N 2015-01a five and four days before the maximum was to confirm that the object is a nova. The only features in these spectra are the emission lines of $\mathrm{H} \alpha$ with equivalent widths $19 \pm 1 \AA$ and $17 \pm 1 \AA$ on Jan. 16 and 17, respectively, and a Na I doublet absorption. After a careful re-calibration of the wavelength scales (see Kurtenkov et al. 2015a), the measured radial velocities of the $\mathrm{H} \alpha$ emission peaks are $-365 \mathrm{~km} \mathrm{~s}^{-1}$ and $-350 \mathrm{~km} \mathrm{~s}^{-1}$. This agrees well with the velocity of $\sim-370 \pm 50 \mathrm{~km} \mathrm{~s}^{-1}$ estimated by Fabrika et al. (2015) for the part of M 31 where the object is located.

The first SCORPIO spectrum was obtained six days before the maximum brightness. The spectral region covered (4050-5850 $\AA$ ) is dominated by absorption lines of ionized and neutral elements such as Fe II, Ti II, Cr II, Mg II, and Fe I. H $\gamma$ and $\mathrm{H} \delta$ are visible in absorption, while $\mathrm{H} \beta$ absorption is partially filled by an emission component. A comparison of the absorption line spectrum with the Jacoby et al. (1984) library of stellar spectra shows very close similarity with an F5I spectrum. The next SCORPIO spectrum obtained on Jan. 21 more

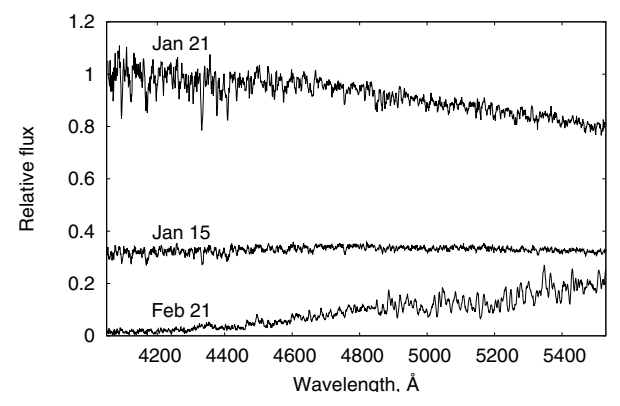

Fig. 3. BTA/SCORPIO spectra obtained with the VPHG1200B and $\mathrm{G}$ grisms. The Jan. 21 spectrum is close to maximum brightness and arbitrarily shifted to unity. The relative flux and shape of the Jan. 15 and Feb. 21 spectra reflect the evolution of the luminosity and colours.

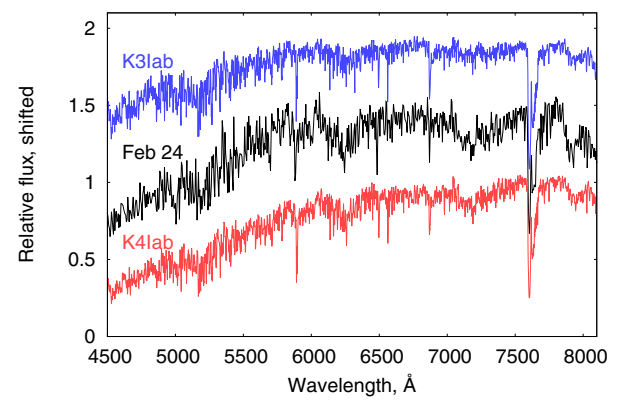

Fig. 4. Combined GTC/OSIRIS spectrum dereddened by $E_{B-V}=$ 0.4 mag in comparison to HD 157999 (K3Iab) and 41 Gem (K4Iab).

or less coincides with the brightness maximum. The absorption line spectrum remained practically the same. However, the continuum is much bluer (Fig. 3) and resembles an F0I spectrum more closely. We cross-correlated these two spectra with an F5I template and found a radial velocity for the pre-maximum spectrum $-462 \pm 38 \mathrm{~km} \mathrm{~s}^{-1}$ and $-534 \pm 14 \mathrm{~km} \mathrm{~s}^{-1}$ for the spectrum around brightness maximum (measured using the IRAF FXCOR package). The cross-correlation of the M31N 2015-01a spectra itself confirms a velocity difference of $64 \pm 13 \mathrm{~km} \mathrm{~s}^{-1}$. Taking into consideration the velocity of $-370 \mathrm{~km} \mathrm{~s}^{-1}$ connected with M31, we found that the heliocentric velocity of the ejected matter on Jan. 15 was $\sim 90 \mathrm{~km} \mathrm{~s}^{-1}$, and it increased to $\sim 160 \mathrm{~km} \mathrm{~s}^{-1}$ on Jan. 21.

One month after the maximum, we obtained two additional SCORPIO spectra in consecutive nights and one OSIRIS spectrum two days later. At that time, the $V$ brightness of the star decreased by $\sim 1.5 \mathrm{mag}$ and the colours drastically reddened. Correspondingly, the spectrum of M31N 2015-01a moved to the lower temperature classes. Now, the neutral element absorptions dominate the spectrum together with weak $\mathrm{TiO}$ molecular bands. Among the strongest absorptions in the spectrum are the Na I doublet, Ba II $6142 \AA$, and $6497 \AA$ Ca I $6573 \AA$ confirmed by Williams et al. (2015), and the near-IR triplet of Ca II. The $\mathrm{Ca}$ II $\mathrm{H} \& \mathrm{~K}$ lines are detected in emission. The strong $\mathrm{Na}$ I lines are clearly split in our OSIRIS spectrum. This allowed us to estimate their $F W H M \sim 200 \pm 15 \mathrm{~km} \mathrm{~s}^{-1}$ and their radial velocity to $\sim-570 \pm 15 \mathrm{~km} \mathrm{~s}^{-1}$. This agrees well with the velocity measured in all our February spectra of M31N 2015-01a (see below) and testifies to the stellar origin of these absorptions. $\mathrm{H} \alpha$ presents as a very weak emission in the OSIRIS spectrum that is divided into two components by a central absorption. In the SCORPIO spectrum only a marginal emission component can be seen. A comparison of the M31N 2015-01a spectrum about one month after the brightness maximum with the stellar spectra libraries of Jacoby et al. (1984) and Le Borgne et al. (2003) shows closest 
similarity with a K3-4I spectral type. By cross-correlating the February spectra with a K3I template, we estimated an average velocity $-588 \pm 40 \mathrm{~km} \mathrm{~s}^{-1}$, which suggests an increase of the ejected matter velocity to $\sim 220 \mathrm{~km} \mathrm{~s}^{-1}$. The cross-correlation of the SCORPIO spectra with that of OSIRIS shows small velocity differences of $8.4 \pm 6.6 \mathrm{~km} \mathrm{~s}^{-1}$ and $-10 \pm 14 \mathrm{~km} \mathrm{~s}^{-1}$ for Feb. 21 and 22, respectively. All uncertainties are $1 \sigma$ equivalent.

Using the task FITSPEC in the STSDAS SYNPHOT ${ }^{3}$ and template spectra from Jacoby et al. (1984), Sánchez-Blázquez et al. (2006) and Le Borgne et al. (2003), we fitted our spectra in an attempt to estimate the reddening. Fitting the spectrum on Jan. 15 with an F5I template, we obtained a reddening $E_{B-V}=0.37$ mag. For the spectrum on Jan. 21 fitted with an F0I template, the corresponding $E_{B-V}$ was 0.33 mag. The merged spectra on Feb. 21 and 22 and the spectrum on Feb. 24 (Fig. 4) were best fitted with a K3I template and $E_{B-V}=0.25 \mathrm{mag}$ and $E_{B-V}=0.42 \mathrm{mag}$, respectively.

Combining the column hydrogen densities of Nieten et al. (2006) with the gas-to-dust (Nedialkov et al. 2011) ratio yields a total colour excess on the line of sight of $E_{B-V}=0.44 \pm$ $0.08 \mathrm{mag}$. An identical result of $E_{B-V}=0.42 \pm 0.03 \mathrm{mag}$ is derived from the maps of the M 31 dust surface density (Draine et al. 2014). A comparison of the $B-V$ and $V-R$ colours with the catalogue compiled by Worthey \& Lee (2011) shows that the reddening $E_{B-V}$ is in the range of $0.15-0.58 \mathrm{mag}$. The mean reddening value, obtained from the spectra $-E_{B-V}=0.35 \pm$ $0.10 \mathrm{mag}$, of which $\sim 0.05 \mathrm{mag}$ can be attributed to the interstellar reddening in our Galaxy (Schlafly \& Finkbeiner 2011), is well within this range. It suggests a true colour of $(B-V)_{0}=0.15 \pm$ $0.10 \mathrm{mag}$ and a $V$-band bolometric correction of +0.03 mag at maximum. Adopting a distance modulus $(m-M)=24.47 \mathrm{mag}^{4}$ and a total-to-selective extinction $R_{V}=3.1$ yields a peak absolute magnitude $M_{V}=-10.13 \pm 0.30 \mathrm{mag}$. This sets the luminosity limits at maximum on $L_{\text {bol }}=8.7_{-2.2}^{+3.3} \times 10^{5} L_{\odot}$. We note that M31N 2015-01a is projected inside a tiny $\left(2^{\prime \prime} \times 2^{\prime \prime}\right)$ HII region \#1527 (Azimlu et al. 2011). Azimlu and collaborators listed an extinction $A_{R}=0.538 \mathrm{mag}$, which corresponds to the somewhat lower $E_{B-V}=0.23 \mathrm{mag}$. An association with this HII region could decrease the luminosity estimate to $\sim 6 \times 10^{5} L_{\odot}$.

\section{Summary}

The outburst of M31N 2015-01a was a highly luminous event, reaching $\sim 10^{6} L_{\odot}$ at the optical maximum. We presented accurate photometry and astrometry for the period January-March 2015. The transient has all the main observational characteristics of red novae, and we conclude that it belongs to this group of events. The fading was slow at first with $t_{2} \sim 50 d$ in $R$ band. In a one-month period, $B-R$ increased by $2.1 \mathrm{mag}$, and the observed spectral type changed from FOI to K3I. The exploration of red novae is so far based on a very small sample of sources, which makes M31N 2015-01a so important: it moves us a step closer to recognizing similarities and differences in their behaviour.

Acknowledgements. This work was partially financed by grant No. BG051 PO001-3.3.06-0057 of the European Social Fund. Based on observations made with the GTC telescope, in the Spanish Observatorio del Roque de los Muchachos of the Instituto de Astrofísica de Canarias, under Director's Discretionary Time. Also based on observations made with the Nordic Optical Telescope at the Observatorio del Roque de los Muchachos. This work makes use of observations from the LCOGT network. K.H. was supported by the project RVO:67985815. A.K. and E.O. gratefully acknowledge observing grant support from the Institute of Astronomy and Rozhen National Astronomical Observatory, Bulgarian Academy of Sciences. K.V. acknowledges support from the Hungarian Research Grant OTKA K-109276, OTKA K-113117 and the "Lendület" Program (LP2012-31) of the Hungarian Academy of Sciences. K.V., K.S. and L.M. have been supported by the Lendület-2009 program of the Hungarian Academy of Sciences and the ESA PECS Contract No. 4000110889/14/NL/NDe. E.A.B. and V.P.G. thank the Russian Foundation for Basic Research for the financial support through Grant 14-02-00759. The research was supported by the Russian Scientific Foundation (grant N 14-5000043). S.F. acknowledges support of the Russian Government Program of Competitive Growth of Kazan Federal University. A.W.S. acknowledges support from NSF grant AST1009566. We thank H. Kučáková, J. Vraštil, and M. Wolf for obtaining images at Ondřejov.

\section{References}

Afanasiev, V. L., \& Moiseev, A. V. 2005, Astron. Lett., 31, 194 Azimlu, M., Marciniak, R., \& Barmby, P. 2011, AJ, 142, 139

Bond, H. E., Henden, A., Levay, Z. G., et al. 2003, Nature, 422, 405 Cepa, J. 1998, Ap\&SS, 263, 369

Dong, S., Kochanek, C. S., Adams, S., \& Prieto, J.-L. 2015, ATel, 7173, 1

Draine, B. T., Aniano, G., Krause, O., et al. 2014, ApJ, 780, 172

Evans, A., Geballe, T. R., Rushton, M. T., et al. 2003, MNRAS, 343, 1054 Fabrika, S., Barsukova, E. A., Valeev, A. F., et al. 2015, ATel, 6985, 1 Goranskij, V. P., Cherjasov, D. V., Safonov, B. S., et al. 2015, ATel, 7206, 1 Hamuy, M., Walker, A. R., Suntzeff, N. B., et al. 1992, PASP, 104, 533 Hamuy, M., Suntzeff, N. B., Heathcote, S. R., et al. 1994, PASP, 106, 566 Hodgkin, S. T., Campbell, H., Fraser, M., et al. 2015, ATel, 6952, 1 Jacoby, G. H., Hunter, D. A., \& Christian, C. A. 1984, ApJS, 56, 257

Jockers, K., Credner, T., Bonev, T., et al. 2000, Kinematika i Fizika Nebesnykh Tel Suppl., 3, 13

Kaminski, T., Menten, K. M., Tylenda, R., et al. 2015, Nature, 520, 322

Kochanek, C. S., Adams, S. M., \& Belczynski, K. 2014, MNRAS, 443, 1319

Kulkarni, S. R., Ofek, E. O., Rau, A., et al. 2007, Nature, 447, 458

Kurtenkov, A., Ovcharov, E., Nedialkov, P., et al. 2015a, ATel, 6941, 1

Kurtenkov, A., Tomov, T., Fabrika, S., et al. 2015b, ATel, 7150, 1

Le Borgne, J.-F., Bruzual, G., Pelló, R., et al. 2003, A\&A, 402, 433

Magnier, E. A., Lewin, W. H. G., van Paradijs, J., et al. 1992, A\&AS, 96, 379

Martini, P., Wagner, R. M., Tomaney, A., et al. 1999, AJ, 118, 1034

Mason, E., Diaz, M., Williams, R. E., Preston, G., \& Bensby, T. 2010, A\&A, 516, A108

Massey, P., Olsen, K. A. G., Hodge, P. W., et al. 2006, AJ, 131, 2478

Munari, U., Henden, A., Kiyota, S., et al. 2002, A\&A, 389, L51

Nedialkov, P., Williams, B., Green, J., \& Hatzidimitriou, D. 2011, in AIP Conf. Ser. 1356, eds. I. Zhelyazkov, \& T. Mishonov, 45

Nieten, C., Neininger, N., Guélin, M., et al. 2006, A\&A, 453, 459

Oke, J. B. 1990, AJ, 99, 1621

Rau, A., Kulkarni, S. R., Ofek, E. O., \& Yan, L. 2007, ApJ, 659, 1536

Rich, R. M., Mould, J., Picard, A., Frogel, J. A., \& Davies, R. 1989, ApJ, 341, L51

Roeser, S., Demleitner, M., \& Schilbach, E. 2010, AJ, 139, 2440

Sánchez-Blázquez, P., Peletier, R. F., Jiménez-Vicente, J., et al. 2006, MNRAS, 371, 703

Schlafly, E. F., \& Finkbeiner, D. P. 2011, ApJ, 737, 103

Shumkov, V., Lipunov, V., Gorbovskoy, E., et al. 2015, ATel, 6911, 1

Sparks, W. B., Bond, H. E., Cracraft, M., et al. 2008, AJ, 135, 605

Tylenda, R., \& Soker, N. 2006, A\&A, 451, 223

Tylenda, R., Hajduk, M., Kamiński, T., et al. 2011, A\&A, 528, A114

Williams, S. C., Darnley, M. J., Bode, M. F., \& Steele, I. A. 2015, ApJL, submitted [arXiv: 1504.07747]

Worthey, G., \& Lee, H.-c. 2011, ApJS, 193, 1

Page 5 is available in the electronic edition of the journal at http: //www . aanda. org

\footnotetext{
3 STSDAS is a product of the Space Telescope Science Institute, which is operated by AURA for NASA.

4 The NED database (http://ned.ipac. caltech. edu/) lists a median $(m-M)=24.45$ mag for M31. If the object lies in the M31 plane, its location suggests a +0.02 mag difference from the centre.
} 
Table 4. Calculated magnitudes for M31N 2015-01a in JohnsonCousins BVRI filters.

\begin{tabular}{|c|c|c|c|c|c|}
\hline JD-2 457000 & Filter & mag & err & Telescope & Airmass \\
\hline 36.264 & $B$ & 19.641 & 0.161 & $\mathrm{RSCH}$ & 1.17 \\
\hline 38.248 & $B$ & 17.046 & 0.017 & BTA & 1.30 \\
\hline 38.345 & $B$ & 16.992 & 0.029 & $\mathrm{RSCH}$ & 1.69 \\
\hline 39.261 & $B$ & 16.549 & 0.017 & $\mathrm{RSCH}$ & 1.19 \\
\hline 39.413 & $B$ & 16.498 & 0.029 & UL50 & 1.53 \\
\hline 40.295 & $B$ & 16.215 & 0.017 & $\mathrm{RSCH}$ & 1.35 \\
\hline 44.196 & $B$ & 15.934 & 0.017 & BTA & 1.16 \\
\hline 46.612 & $B$ & 16.109 & 0.026 & LCOGT & 1.32 \\
\hline 48.658 & $B$ & 16.679 & 0.242 & LCOGT & 1.71 \\
\hline 51.611 & $B$ & 17.261 & 0.026 & LCOGT & 1.38 \\
\hline 60.323 & $B$ & 17.865 & 0.096 & $\mathrm{KSCH}$ & 1.79 \\
\hline 61.252 & $B$ & 17.974 & 0.026 & $\mathrm{KSCH}$ & 1.32 \\
\hline 67.257 & $B$ & 18.294 & 0.066 & $\mathrm{KSCH}$ & 1.42 \\
\hline 68.246 & $B$ & 18.383 & 0.022 & $\mathrm{RSCH}$ & 1.53 \\
\hline 72.249 & $B$ & 18.719 & 0.103 & $\mathrm{KSCH}$ & 1.46 \\
\hline 75.224 & $B$ & 18.791 & 0.018 & BTA & 1.92 \\
\hline 93.340 & $B$ & 20.076 & 0.051 & NOT & 2.53 \\
\hline 96.346 & $B$ & 20.385 & 0.041 & NOT & 2.99 \\
\hline 38.246 & $\bar{V}$ & 16.416 & 0.008 & BTA & 1.29 \\
\hline 44.187 & V & 15.436 & 0.011 & BTA & 1.14 \\
\hline 66.319 & V & 16.626 & 0.012 & $\mathrm{KSCH}$ & 1.93 \\
\hline 67.243 & V & 16.621 & 0.026 & $\mathrm{KSCH}$ & 1.35 \\
\hline 72.241 & V & 16.832 & 0.065 & $\mathrm{KSCH}$ & 1.41 \\
\hline 75.225 & V & 16.873 & 0.013 & BTA & 1.93 \\
\hline 89.323 & V & 17.869 & 0.059 & UL50 & 1.92 \\
\hline 92.246 & V & 18.098 & 0.108 & $\mathrm{KSCH}$ & 1.91 \\
\hline 36.243 & $R$ & 18.087 & 0.026 & $\mathrm{RSCH}$ & 1.11 \\
\hline 37.210 & $R$ & 17.213 & 0.011 & $\mathrm{R} 2 \mathrm{M}$ & 1.05 \\
\hline 38.246 & $R$ & 16.051 & 0.011 & BTA & 1.29 \\
\hline 38.330 & $R$ & 16.047 & 0.009 & $\mathrm{RSCH}$ & 1.55 \\
\hline 39.242 & $R$ & 15.679 & 0.009 & $\mathrm{RSCH}$ & 1.13 \\
\hline 40.283 & $R$ & 15.407 & 0.009 & $\mathrm{RSCH}$ & 1.29 \\
\hline 40.390 & $R$ & 15.364 & 0.012 & $\mathrm{R} 2 \mathrm{M}$ & 2.38 \\
\hline 44.189 & $R$ & 15.133 & 0.011 & BTA & 1.14 \\
\hline 46.617 & $R$ & 15.165 & 0.013 & LCOGT & 1.34 \\
\hline 48.661 & $R$ & 15.347 & 0.032 & LCOGT & 1.75 \\
\hline 51.615 & $R$ & 15.609 & 0.011 & LCOGT & 1.41 \\
\hline 57.285 & $R$ & 15.678 & 0.011 & ONDR & 1.36 \\
\hline 59.238 & $R$ & 15.692 & 0.011 & ONDR & 1.20 \\
\hline 60.317 & $R$ & 15.675 & 0.012 & $\mathrm{KSCH}$ & 1.73 \\
\hline 61.234 & $R$ & 15.683 & 0.011 & $\mathrm{KSCH}$ & 1.24 \\
\hline 62.596 & $R$ & 15.681 & 0.009 & LCOGT & 1.50 \\
\hline 64.608 & $R$ & 15.689 & 0.009 & LCOGT & 1.66 \\
\hline 66.310 & $R$ & 15.725 & 0.011 & $\mathrm{KSCH}$ & 1.82 \\
\hline 67.239 & $R$ & 15.741 & 0.009 & $\mathrm{KSCH}$ & 1.33 \\
\hline 68.230 & $R$ & 15.804 & 0.013 & $\mathrm{RSCH}$ & 1.42 \\
\hline 72.244 & $R$ & 15.839 & 0.027 & $\mathrm{KSCH}$ & 1.42 \\
\hline 73.250 & $R$ & 15.858 & 0.009 & ONDR & 1.40 \\
\hline 75.223 & $R$ & 15.894 & 0.024 & BTA & 1.90 \\
\hline 80.270 & $R$ & 16.111 & 0.011 & ONDR & 1.68 \\
\hline 86.329 & $R$ & 16.417 & 0.021 & ONDR & 2.72 \\
\hline 87.302 & $R$ & 16.437 & 0.016 & ONDR & 2.28 \\
\hline 89.266 & $R$ & 16.642 & 0.012 & ONDR & 1.88 \\
\hline 89.337 & $R$ & 16.617 & 0.061 & UL50 & 2.08 \\
\hline 92.245 & $R$ & 16.987 & 0.104 & $\mathrm{KSCH}$ & 1.90 \\
\hline 93.345 & $R$ & 17.011 & 0.011 & NOT & 2.68 \\
\hline 96.351 & $R$ & 17.315 & 0.013 & NOT & 3.19 \\
\hline 99.283 & $R$ & 17.617 & 0.035 & ONDR & 2.54 \\
\hline 105.279 & $R$ & 18.499 & 0.084 & ONDR & 2.79 \\
\hline
\end{tabular}

Table 4. continued.

\begin{tabular}{cccccc}
\hline \hline JD-2 457000 & Filter & mag & err & Telescope & Airmass \\
\hline 60.320 & $I$ & 15.028 & 0.021 & KSCH & 1.76 \\
61.245 & $I$ & 15.017 & 0.018 & KSCH & 1.28 \\
67.241 & $I$ & 15.019 & 0.018 & KSCH & 1.34 \\
68.272 & $I$ & 15.085 & 0.016 & RSCH & 1.78 \\
72.246 & $I$ & 15.048 & 0.028 & KSCH & 1.44 \\
89.357 & $I$ & 15.344 & 0.111 & UL50 & 2.34 \\
92.248 & $I$ & 15.377 & 0.065 & KSCH & 1.92 \\
93.350 & $I$ & 15.557 & 0.021 & NOT & 2.84 \\
96.356 & $I$ & 15.735 & 0.021 & NOT & 3.42 \\
\hline
\end{tabular}

